\title{
Simon Bouquet (ed.), Les genres de la parole
}

\section{Elettra Bordino Zorzi}

\section{(2) OpenEdition}

\section{Journals}

\section{Édition électronique}

URL : http://journals.openedition.org/studifrancesi/36868

DOI : $10.4000 /$ studifrancesi.36868

ISSN : 2427-5856

\section{Éditeur}

Rosenberg \& Sellier

\section{Édition imprimée}

Date de publication : 1 juillet 2005

Pagination : 212-213

ISSN : 0039-2944

\section{Référence électronique}

Elettra Bordino Zorzi, «Simon Bouquet (ed.), Les genres de la parole», Studi Francesi [En ligne], 145 (XLIX II) | 2005, mis en ligne le 30 novembre 2015, consulté le 18 avril 2021. URL : http:// journals.openedition.org/studifrancesi/36868; DOI : https://doi.org/10.4000/studifrancesi.36868

\section{Ce document a été généré automatiquement le 18 avril 2021.}

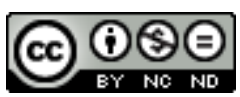

Studi Francesi è distribuita con Licenza Creative Commons Attribuzione - Non commerciale - Non opere derivate 4.0 Internazionale. 


\title{
Simon Bouquet (ed.), Les genres de la parole
}

\author{
Elettra Bordino Zorzi
}

\section{RÉFÉRENCE}

SIMON BOUQUET (ed.), Les genres de la parole, «Langages», 153, mars 2004, pp. 128.

Dans l'introduction Linguistique générale et linguistique des genres (pp. 3-14), Simon BOUQUET met ce numéro de «Langages» sous le signe d'une réflexion épistémologique visant à une description consensuelle du sens, objet principal des sciences du langage, ce à quoi contribuerait d'une manière fondamentale le concept de genre. Celui-ci, en effet, permet d'articuler la linguistique de la parole, dont il relève, à celle de la langue, car il se présente comme à la fois l'actualisation et la détermination supra-segmentale des signifiés grammaticaux des phrases. Aussi parvient-il à conjuguer la tradition rhétorique-herméneutique attentive au contexte et les théories logico-grammaticales dont il dément toutefois le principe de la compositionalité de la signification. La première des trois sections de la revue, consacrée aux genres de l'oral, s'ouvre sur l'essai de Mary-Annick MOREL, Intonation, Regard et Genres dans le dialogue à bâtons rompus (pp. 15-27). Les variations de mélodie et d'intensité de la voix du locuteur ainsi que les déplacements de son regard constituent ici les paramètres guidant la subdivision en paragraphes et l'analyse d'un dialogue entre deux étudiantes, dont la caractéristique principale s'avère être l'extrême variabilité générique. Dans Sémiotique grammaticale et sémantique des (genres de) jeux de langage: les «pronoms personnels clitiques» en français (pp. 28-40), Simon BOUQUET, après avoir érigé le sens en objet empirique définissable par voie différentielle, distingue deux niveaux descriptifs qu'il va appliquer à l'étude des pronoms personnels clitiques en français: le sémiotique (grammatical) et le sémantique (interprétatif). Au premier revient l'identification des traits syntagmatiques, morphématiques et phonématiques et de leur triple valeur sémiotique - iconique, indexicale, symbolique selon Peirce; quant au second, il porte sur les traits génériques - 
p. e. politesse, hypocoristique caressant, agressivité - permettant de désambiguïser des énoncés homonymiques.

L'article de Catherine KEBRAT-ORECCHIONI et de Véronique TRAVERSO (Types d'Interactions et genres de l'oral, pp. 41-51) ramène à l'unité les divergences terminologiques et/ou typologiques qui affectent le champ hétérogène des échanges verbaux, en postulant l'existence de deux sortes de genres: les G1, catégories de discours qu'opposent des critères externes, à savoir situationnels, et les $\mathrm{G} 2$, fondés au contraire sur des critères internes de nature linguistique et énonciative. Dans la pratique discursive, ces derniers se combinent librement pour former un G1, au nom d'une organisation métissée et floue, souvent sujette à négociation, dont rend ici compte l'examen d'un extrait de conversation. Différente est la perspective, nettement psycolinguistique, qu'adoptent Ioanna BERTHOUD-PAPANDROPOULOU et Helga KILCHER dans Acquisition du langage et genres énonciatifs (pp. 52-61). Sur la base de deux recherches expérimentales, ces spécialistes se fixent pour but de démontrer que la compétence conversationnelle de l'enfant, et notamment sa maîtrise des genres comme, dans le cas présent, celui de la transmission d'un message et celui de la détection et réparation d'un malentendu, se développe par étapes successives à partir d'un type primaire: le dialogue à deux qu'enseigne la mère. Avec les trois contributions suivantes, le débat se focalise sur les constellations génériques de l'écrit. Jean-Michel ADAM et Ute HEIDMANN (Des genres à la généricité. L'exemple des contes (Perrault et les Grimm), pp. 62-72) se penchent sur des textes qui sont considérés comme l'archétype fondateur du genre du conte (Perrault en France et les frères Grimm en Allemagne) pour conclure que, loin de répondre à un schéma fixe, leur constitution est tellement instable et complexe qu'il faut, par un saut conceptuel, parler plutôt de "généricité». Celle-ci se situe à l'intersection de trois instances énonciatives - auctoriale, éditoriale, lectoriale - et prend place, à côté de la textualité et de la transtextualité, dans un modèle dynamique et comparatif des productions discursives. Le travail suivant (Denise MALRIEU, Linguistique de corpus, genres textuels, temps et personnes, pp. 73-85) exploite un corpus d'œuvres narratives du $\mathrm{XX}^{\mathrm{e}}$ siècle en partie balisées pour tâcher d'identifier, suivant une approche multivariée, les traits morphosyntaxiques - en particulier temps des verbes et occurrences des personnes susceptibles de marquer efficacement les genres. Dans l'essai de Bénédicte FACQUES et de Carol SANDERS (Textes journalistiques et analyse contrastive du genre en didactique, pp. 86-97), en revanche, les typologies de textes journalistiques et les thèses fonctionnalistes de Halliday et de Wilkins ne sont convoquées que pour enrichir l'enseignementapprentissage des langues étrangères d'une compétence nouvelle, qui se révèle précieuse dans une optique contrastive: la connaissance des conventions génériques non seulement aide à la compréhension mutuelle mais encore initie à la différence culturelle.

3 Le volume se termine par les Questions épistémologiques de la troisième section. Dans le cadre d'une conceptualisation de l'agir humain, Jean-Paul BRONCKART (Les genres de textes et leur contribution au développement psychologique, pp. 98-108) renverse les termes d'un binôme largement partagé dans ce secteur linguistique en opposant les genres de textes, qu'il considère comme des unités ccommunicatives globales fortement tributaires du contexte social, aux types de discours. Ceux-ci correspondent aux fragments linguistiques dont les premiers sont composés et où se réalise l'interconnexion du collectif et de l'individuel indispensable à l'épanouissement psychologique de toute personne. L'article de Jean-Claude BEACCO, intitulé Trois 
perspectives linguistiques sur la notion de genre (pp. 109-119), décline la notion de genre discursif suivant trois points de vue méthodologiques à son avis complémentaires: celui des représentations métalinguistiques ordinaires du locuteur naif, celui de la linguistique textuelle avec ses régularités difficilement saisissables et ses cristallisations labiles et à géométrie variable, celui enfin de l'analyse du discours qui inscrit, on le sait, des entités verbales dans un lieu envisagé sous son aspect social, dans une spatialité qui finit par la «communauté de communication» théorisée par les ethnographes. D'après F. RASTIER (Poétique et textualité, pp. 120-126), la poétique, traditionnellement confinée dans le domaine littéraire, peut au contraire donner son apport à la conception de la textualité, une fois qu'elle s'impose comme linguistique des genres. Dans sa sémiosis le texte con figure le genre au moins autant qu'il en est configuré, dans un jeu de renvois visibles au sein d'un corpus, et ce faisant il se conforme, par delà les règles impératives de la langue, aux normes flexibles et socialisées de la parole. 